\title{
Classification problems in 2-representation theory
}

\author{
Volodymyr Mazorchuk ${ }^{1}$
}

Published online: 5 April 2017

(C) The Author(s) 2017. This article is an open access publication

\begin{abstract}
This article surveys recent advances and future challenges in the 2representation theory of finitary 2-categories with a particular emphasis on problems related to classification of various classes of 2-representations.
\end{abstract}

Keywords Categorification · 2-Category · 2-Representation · Simple transitive 2-representation $\cdot$ Classification

\section{Introduction}

Higher representation theory emerged as an offspring of categorification. The latter term traditionally describes the approach, originated in $[12,13]$, of upgrading settheoretical notions to category theoretical with a hope to create more structure. The major breakthrough of categorification was invention of Khovanov homology in [28]. After that several other spectacular applications followed, for example, to Broué's abelian defect group conjecture in [11], to study of modules over Lie superalgebras in $[7,8]$ and to various other problems. The common feature of all these and many other applications is that construction and comparison of functorial actions on different categories was the key part of the argument.

Functorial actions form the red thread of [3] leading to an alternative reformulation of Khovanov homology in [54] using BGG category $\mathcal{O}$. In [11] and, later on, in [50], functorial actions were abstractly reformulated in terms of representation theory of certain 2-categories. This direction of study was subsequently called higher representation theory or, alternatively, just 2-representation theory to emphasize that, so far,

\footnotetext{
$\bowtie$ Volodymyr Mazorchuk mazor@math.uu.se

1 Department of Mathematics, Uppsala University, Box. 480, 75106 Uppsala, Sweden
} 
it directs only at this second level of general higher categories. However, one has to note that these and many further papers like [15,29] and other mainly study special examples of 2-categories which originate in topologically motivated diagrammatic calculus.

The series [40-45] of papers took higher representation theory to a more abstract level. These papers started a systematic study of abstract 2-analogues of finite dimensional algebras, called finitary 2-categories, and their representation theory. Despite of the fact that the 2-category of 2-representations of an abstract finitary 2-category is much more complicated than the category of modules over a finite dimensional algebra (in particular, it is not abelian), it turned out that, in many cases, it is possible to construct, compare and even classify various classes of 2-representations. These kinds of problems were recently studied in a number of papers, see [32,37-39,44-48,58]. The aim of the present article is to give an overview of these results with a particular emphasis on open problems and future challenges.

We start in Sect. 2 with a brief description of main objects of study. Section 3 lists a number of classical examples. Sections 4, 5 and 6 then concentrate on special classes of 2-representations. In particular, Sect. 4 addresses cell 2-representations and Sect. 5 is devoted to simple transitive 2-representations. We do not provide any proofs but rather give explicit references to original sources.

The present paper might serve as a complement reading to the series of lectures on higher representation theory which the author gave during Brazilian Algebra Meeting in Diamantina, Brazil, in August 2016.

\section{Finitary 2-categories and their 2-representations}

\subsection{Finitary 2-categories}

A 2-category is a category which is enriched over the monoidal category Cat of small categories (the monoidal structure of the latter category is given by Cartesian product). This means that a 2-category $\mathscr{C}$ consists of

- objects, denoted i, j, k,..;

- small morphism categories $\mathscr{C}(i, j)$, for all $i, j \in \mathscr{C}$;

- bifunctorial compositions $\mathscr{C}(j, \mathrm{k}) \times \mathscr{C}(\mathrm{i}, \mathrm{j}) \rightarrow \mathscr{C}(\mathrm{i}, \mathrm{k})$, for all $\mathrm{i}, \mathrm{j}, \mathrm{k} \in \mathscr{C}$;

- and identity objects $\mathbb{1}_{i} \in \mathscr{C}(i, i)$, for all $i \in \mathscr{C}$;

which satisfy the obvious collection of strict axioms. The following terminology is standard:

- objects in $\mathscr{C}(i, j)$ are called 1-morphisms of $\mathscr{C}$ and will be denoted by F, G, etc.;

- morphisms in $\mathscr{C}(i, j)$ are called 2-morphisms of $\mathscr{C}$ and will be denoted by $\alpha, \beta$, etc.;

- composition of 2-morphisms inside $\mathscr{C}(i, j)$ is called vertical and will be denoted by $\circ_{v}$

- composition of 2-morphisms coming from $\mathscr{C}(j, k) \times \mathscr{C}(i, j) \rightarrow \mathscr{C}(i, k)$ is called horizontal and will be denoted by $\circ_{h}$. 
As usual, for a 1-morphism $\mathrm{F}$, the identity 2-morphism for $\mathrm{F}$ is denoted $\mathrm{id}_{\mathrm{F}}$, moreover, for a 2-morphism $\alpha$, the compositions $\operatorname{id}_{\mathrm{F}} \circ_{h} \alpha$ and $\mathrm{id}_{\mathrm{F}} \circ_{h} \alpha$ are denoted by $\mathrm{F}(\alpha)$ and $\alpha_{\mathrm{F}}$, respectively. We refer the reader to $[34,36]$ for more details on 2-categories and for various generalizations, in particular, for the corresponding non-strict notion of a bicategory.

Let $\mathbb{k}$ be an algebraically closed field. Recall that a category $\mathcal{C}$ is called finitary $\mathbb{k}$-linear provided that it is equivalent to the category of projective modules over a finite dimensional (associative) $\mathbb{k}$-algebra. Each such category is $\mathbb{k}$-linear, that is enriched over the category of $\mathbb{k}$-vector spaces, moreover, it is idempotent split and Krull-Schmidt and has finitely many isomorphism classes of indecomposable objects and finite dimensional (over $\mathbb{k}$ ) spaces of morphisms.

Following [40, Subsection 2.2], we will say that a 2-category $\mathscr{C}$ is finitary over $\mathbb{k}$ provided that

- each $\mathscr{C}(i, j)$ is finitary $\mathbb{k}$-linear;

- all compositions are biadditive and $\mathbb{k}$-bilinear, whenever appropriate;

- all $\mathbb{1}_{i}$ are indecomposable.

The last condition is a technical condition which makes the life easier at many occasions. From the representation theoretic prospective, this condition is not restrictive as, starting from a 2-category satisfying all other conditions, one can use idempotent splitting to produce a finitary 2-category with essentially the same representation theory.

In what follows, we will simply say that $\mathscr{C}$ is finitary as our field $\mathbb{k}$ will be fixed throughout the paper (with the exception of examples related to Soergel bimodules where $\mathbb{k}=\mathbb{C}$ ).

\subsection{2-representations}

For two 2-categories $\mathscr{A}$ and $\mathscr{C}$, a 2-functor $\Phi: \mathscr{A} \rightarrow \mathscr{C}$ is a functor which respects all 2-categorical structure. This means that $\Phi$

- maps 1-morphisms to 1-morphisms;

- maps 2-morphisms to 2-morphisms;

- is compatible with composition of 1-morphisms;

- is compatible with both horizontal and vertical composition of 2-morphisms;

- sends identity 1-morphisms to identity 1-morphisms;

- sends identity 2-morphisms to identity 2-morphisms.

A 2-representation of a 2-category $\mathscr{C}$ is a 2-functor to some fixed 2-category. Classical examples of such target 2-categories are:

- the 2-category Cat of small categories, here 1-morphisms are functors and 2morphisms are natural transformations;

- the 2-category $\mathfrak{A}_{\mathbb{k}}^{f}$ of finitary $\mathbb{k}$-linear categories, here 1-morphisms are additive $\mathbb{k}$-linear functors and 2-morphisms are natural transformations;

- the 2-category $\mathfrak{R}_{\mathbb{k}}$ of finitary abelian $\mathbb{k}$-linear categories, here objects are categories equivalent to module categories of finite dimensional (associative) $\mathbb{k}$ - 
algebras, 1-morphisms are right exact additive $\mathbb{k}$-linear functors and 2-morphisms are natural transformations.

All 2-representations of a 2-category $\mathscr{C}$ (in a fixed target 2-category) form a 2category. In this 2-category we have:

- 1-morphisms are (strong) 2-natural transformations;

- 2-morphisms are modifications.

One has to make a choice for the level of strictness for 1-morphisms in the 2-category of 2-representations of $\mathscr{C}$. In the language of [34, Subsection 1.2], this corresponds to choosing between the so-called strong or strict transformations. Strict transformations were considered in the paper [40] and the setup was changed to strong transformations in [42]. The latter allows for more flexibility and more reasonable results (for example, the relation of equivalence of two 2-representations becomes symmetric).

For a finitary 2-category $\mathscr{C}$, its 2-representations in $\mathfrak{A}_{\mathbb{k}}^{f}$ are called finitary additive 2-representations and the corresponding 2-category of 2-representations is denoted by $\mathscr{C}$ - afmod. Further, 2-representations of $\mathscr{C}$ in $\mathfrak{R}_{\mathbb{k}}$ are called abelian 2-representations and the corresponding 2-category of 2-representations is denoted by $\mathscr{C}-\bmod$. Note that neither $\mathscr{C}-$ afmod nor $\mathscr{C}-\bmod$ are abelian categories.

We will usually denote 2 -representations of $\mathscr{C}$ by $\mathbf{M}, \mathbf{N}$ etc. For the sake of readability, it is often convenient to use the actions notation $\mathrm{F} X$ instead of the representation notation $\mathbf{M}(\mathrm{F})(X)$.

Here is an example of a 2-representation: for $i \in \mathscr{C}$, the principal 2-representation $\mathbf{P}_{i}$ is defined to be the Yoneda 2-representation $\mathscr{C}\left(i,{ }_{-}\right)$. If $\mathscr{C}$ is finitary, we have $\mathbf{P}_{i} \in \mathscr{C}$-afmod.

Two 2-representations $\mathbf{M}$ and $\mathbf{N}$ of $\mathscr{C}$ are called equivalent provided that there is a 2-natural transformation $\Phi: \mathbf{M} \rightarrow \mathbf{N}$ such that $\Phi_{i}$ is an equivalence of categories, for each $i \in \mathscr{C}$.

\subsection{Abelianization}

Given a finitary $\mathbb{k}$-linear category $\mathcal{C}$, the diagrammatic abelianization of $\mathcal{C}$ is the category $\overline{\mathcal{C}}$ of diagrams of the form $X \stackrel{\alpha}{\longrightarrow} Y$ over $\mathcal{C}$ with morphisms being the obvious commutative squares modulo the projective homotopy relations. The category $\overline{\mathcal{C}}$ is abelian and is equivalent to the category of modules over a finite dimensional $\mathbb{k}$ algebra. The original category $\mathcal{C}$ canonically embeds into $\overline{\mathcal{C}}$ via diagrams of the form $0 \rightarrow Y$ and this embedding provides an equivalence between $\mathcal{C}$ and the category of projective objects in $\overline{\mathcal{C}}$. We refer the reader to [17] for details.

For a finitary 2-category $\mathscr{C}$, using diagrammatic abelianization and component-wise action on diagrams defines a 2 -functor

$$
\div: \mathscr{C} \text {-afmod } \rightarrow \mathscr{C}-\bmod
$$

called abelianization, see [40, Subsection 3.1]. In [38, Section 3] one finds a more advanced refinement of this construction which is way more technical but also has some extra nice properties. 


\subsection{Fiat 2-categories}

As we will see, many examples of finitary 2-categories have additional structure which plays very important role and significantly simplifies many arguments. This additional structure, on a finitary 2-category $\mathscr{C}$, consists of

- a weak involution $\star$ which inverts the direction of both 1- and 2-morphisms,

- adjunction morphisms $\varepsilon^{(\mathrm{F})}: \mathrm{F} \circ \mathrm{F}^{\star} \rightarrow \mathbb{1}_{j}$ and $\eta^{(\mathrm{F})}: \mathbb{1}_{i} \rightarrow \mathrm{F}^{\star} \circ \mathrm{F}$, for each $\mathrm{F} \in \mathscr{C}(i, j)$, which make $\left(\mathrm{F}, \mathrm{F}^{\star}\right)$ a pair of adjoint 1-morphisms in the sense that

$$
\operatorname{id}_{\mathrm{F}}=\varepsilon_{\mathrm{F}}^{(\mathrm{F})} \circ_{v} \mathrm{~F}\left(\eta^{(\mathrm{F})}\right) \quad \text { and } \quad \operatorname{id}_{\mathrm{F}}^{\star}=\mathrm{F}^{\star}\left(\varepsilon^{(\mathrm{F})}\right) \circ_{v} \eta_{\mathrm{F}^{\star}}^{(\mathrm{F})}
$$

A 2-category $\mathscr{C}$ having such an additional structure is called fiat, where "f" stands for finitary, "i" stands for involution, "a" stands for adjunction and "t" stands for 2category, see [40, Subsection 2.4]. If a similar structure exists for a not necessarily involutive anti-autoequivalence $\star$, the 2 -category $\mathscr{C}$ is called weakly fiat, see [41, Subsection 7.3] and [45, Appendix].

In many situations, involutions in 2-categories change the direction of 1-morphisms but preserve the direction of 2-morphisms, see e.g. [34, Page 3]. The above definition, in which both the directions of 1- and 2-morphisms get reversed, is motivated by the 2category of endofunctors of $A$-mod, for a finite dimensional $\mathbb{k}$-algebra $A$. For each pair ( $\mathrm{F}, \mathrm{G})$ of adjoint endofunctors of $A$-mod, there is an $A$ - $A$-bimodule $Q$ such that $\mathrm{F}$ is isomorphic to $Q \otimes_{A-}$ and $\mathrm{G}$ is isomorphic to $\operatorname{Hom}_{A-}\left(Q,{ }_{-}\right)$, see [1, Chapter I]. Natural transformations between functors correspond to homomorphisms between bimodules. When taking the adjoint functor, the bimodule $Q$ ends up on the contravariant place of the bifunctor Hom and hence the direction of natural transformations gets reversed.

In the literature one could find similar structures under the name of rigid tensor categories categories, see e.g. [14].

\subsection{Grothendieck decategorification}

For an finitary $\mathbb{k}$-linear category $\mathcal{C}$, let $[\mathcal{C}]_{\oplus}$ denote the split Grothendieck group of $\mathcal{C}$. Then $[\mathcal{C}]_{\oplus}$ is a free abelian group which has a canonical generating set given by isomorphism classes of indecomposable objects in $\mathcal{C}$. For a category $\mathcal{C}$ equivalent to $A$-mod, for some finite dimensional $\mathbb{k}$-algebra $A$, let $[\mathcal{C}]$ denote the Grothendieck group of $\mathcal{C}$. Then $[\mathcal{C}]$ is a free abelian group which has a canonical generating set given by isomorphism classes of simple objects in $\mathcal{C}$.

Let $\mathscr{C}$ be a finitary 2-category and $\mathbf{M} \in \mathscr{C}$-afmod. Let $[\mathscr{C}]_{\oplus}$ denote the ordinary category which has the same objects as $\mathscr{C}$ and in which morphisms are given by $[\mathscr{C}]_{\oplus}(i, j):=[\mathscr{C}(i, j)]_{\oplus}$ with induced composition. The category $[\mathscr{C}]_{\oplus}$ is called the Grothendieck decategorification of $\mathscr{C}$. The category $[\mathscr{C}]_{\oplus}$ acts on abelian groups $[\mathbf{M}(i)]_{\oplus}$, where $i \in \mathscr{C}$, which in this way defines the Grothendieck decategorification $[\mathbf{M}]_{\oplus}$ of $\mathbf{M}$.

If $\mathscr{C}$ is fiat and $\mathbf{M} \in \mathscr{C}$-mod, then $[\mathscr{C}]_{\oplus}$ acts on abelian groups $[\mathbf{M}(i)]$, where $i \in \mathscr{C}$, which in this way defines the Grothendieck decategorification $[\mathbf{M}]$ of $\mathbf{M}$. 
We note that there are alternative decategorifications, notably, the trace decategorification introduced in [2].

\section{Examples of finitary 2-categories}

\subsection{Set-theoretic issues}

There are some set-theoretic complications due to the fact that, by definition, each $\mathscr{C}(i, j)$ of a 2 -category $\mathscr{C}$ has to be small. This prevents us to consider, for example, the category of all $A$ - $A$-bimodules, for a finite dimensional $\mathbb{k}$-algebra $A$, as a 2-category (with one formal object that can be identified with $A$-mod). The reason for that is the observation that the category of all bimodules is not small.

In what follows we will give many examples by considering all endofunctors (of some type) of some category $\mathcal{C}$. To avoid the above problem, we will always assume that $\mathcal{C}$ is small. This, however, creates a choice. For example, one has to choose a small category $\mathcal{C}$ equivalent to $A$-mod. Different choices of $\mathcal{C}$ lead to different, however, usually biequivalent, 2-categories.

\subsection{Projective endofunctors of $\boldsymbol{A}$-mod}

Let $A$ be a finite dimensional, basic, connected $\mathbb{k}$-algebra. Fix a small category $\mathcal{C}$ equivalent to $A$-mod. Recall that a projective $A$ - $A$-bimodule is an $A$ - $A$-bimodule from the additive closure $\operatorname{add}\left(A \otimes_{\mathbb{k}} A\right)$ of ${ }_{A} A \otimes_{\mathbb{k}} A_{A}$. We also have the regular or identity $A$ - $A$-bimodule ${ }_{A} A_{A}$.

Denote by $\mathscr{C}_{A}=\mathscr{C}_{A, \mathcal{C}}$ the 2-category which has

- one object $i$ (which should be though of as $\mathcal{C}$ );

- as 1-morphisms, endofunctors of $\mathcal{C}$ from the additive closure of endofunctors given by tensoring with projective or regular $A$ - $A$-bimodules;

- as 2-morphisms, all natural transformations of functors.

This 2-category appears in [40, Subsection 7.3].

If $A$ is simple, then $A \cong \mathbb{k}$ and $\mathscr{C}_{A}$ has a unique (up to isomorphism) indecomposable 1-morphism, namely $\nVdash_{i}$. If $A$ is not simple, let $e_{1}+e_{2}+\cdots+e_{n}=1$ be a primitive decomposition of the identity $1 \in A$. Then, apart from $\nVdash_{i}$, the 2-category $\mathscr{C}_{A}$ has $n^{2}$ additional indecomposable 1-morphisms $\mathrm{F}_{i j}$, where, for $i, j=1,2, \ldots, n$, the morphism $\mathrm{F}_{i j}$ corresponds to tensoring with $A e_{i} \otimes_{\mathbb{k}} e_{j} A$. We note that

$$
\mathrm{F}_{i j} \circ \mathrm{F}_{s t} \cong \mathrm{F}_{i t}^{\operatorname{dim}\left(e_{j} A e_{s}\right)} \text { and } \mathrm{F} \circ \mathrm{F} \cong \mathrm{F}^{\operatorname{dim}(A)}, \text { for } \mathrm{F}:=\bigoplus_{i, j=1}^{n} \mathrm{~F}_{i j}
$$

The 2-category $\mathscr{C}_{A}$ is always finitary. It is weakly fiat if and only if $A$ is self-injective. It is fiat if and only if $A$ is weakly symmetric. In the latter case, $\left(\mathrm{F}_{i j}, \mathrm{~F}_{j i}\right)$ forms an adjoint pair of 1-morphisms, for all $i$ and $j$. 


\subsection{Finitary 2-categories of all bimodules}

For $n=1,2, \ldots$, let $A_{n}$ denote the $\mathbb{k}$-algebra given as the quotient of the path algebra of the quiver

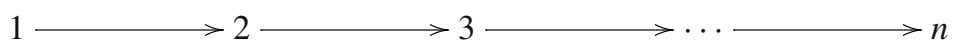

by the relations that the product of any two arrows is zero. Let $\mathcal{C}$ be a small category equivalent to $A$-mod.

Denote by $\mathscr{F}_{A_{n}}=\mathscr{F}_{A_{n}, \mathcal{C}}$ the 2-category which has

- one object $i$ (which should be though of as $\mathcal{C}$ );

- as 1-morphisms, all right exact endofunctors of $\mathcal{C}$;

- as 2-morphisms, all natural transformations of functors.

The 2-category $\mathscr{F}_{A_{n}}$ is finitary, see [48, Section 2]. The reason for that is the fact that the enveloping algebra $A_{n} \otimes_{\mathbb{k}} A_{n}^{\text {op }}$ of $A_{n}$ is a special biserial algebra in the sense of $[9,55]$, so one can use the classification of indecomposable modules for this algebra to check that it has, in fact, only finitely many of them, up to isomorphism. Unless $n=1$, the 2-category $\mathscr{F}_{A_{n}}$ is neither fiat nor weakly fiat.

Note that a similar 2-category $\mathscr{F}_{A}$ can be defined for any connected and basic $\mathbb{k}$-algebra $A$. However, if $\mathscr{F}_{A}$ is fiat, then $A \cong A_{n}$, for some $n$, see [48, Theorem 1].

\subsection{Subbimodules of the identity bimodule}

Let $\Gamma$ be a finite oriented tree and $A$ be the path algebra of $\Gamma$. Let $\mathcal{C}$ be a small category equivalent to $A$-mod.

Denote by $\mathscr{G}_{A}=\mathscr{G}_{A, \mathcal{C}}$ the 2-category which has

- one object $i$ (which should be though of as $\mathcal{C}$ );

- as 1-morphisms, all endofunctors of $\mathcal{C}$ from the additive closure of subfunctors of the identity functor;

- as 2-morphisms, all natural transformations of functors.

The 2-category $\mathscr{G}_{A}$ is finitary due to the fact that the regular $A$ - $A$-bimodule is multiplicity free and hence has only finitely many subbimodules. Unless $\Gamma$ has one vertex, the 2-category $\mathscr{F}_{A_{n}}$ is neither fiat nor weakly fiat.

The 2-category $\mathscr{G}_{A}$ first appeared, slightly disguised, in [23] (inspired by [22]), for $\Gamma$ being the quiver in (2). It was further studied, for various special types of trees, in [24] and [57,58].

\subsection{Soergel bimodules for finite Coxeter systems}

Let $(W, S)$ be a finite Coxeter system and $\mathfrak{h}$ be a reflection faithful complexified $W$ module. Let, further, $\mathrm{C}$ be the corresponding coinvariant algebra, that is the quotient of $\mathbb{C}[\mathfrak{h}]$ by the ideal generated by homogeneous $W$-invariant polynomials of positive 
degree. Then $\mathrm{C}$ is a finite dimensional algebra which carries the natural structure of a regular $W$-module, in particular, $\operatorname{dim}(\mathrm{C})=|W|$.

For $s \in S$, denote by $\mathrm{C}^{s}$ the subalgebra of $s$-invariants in $\mathrm{C}$. Then $\mathrm{C}$ is a free $\mathrm{C}^{S}$ module of rank two. Both algebras $\mathrm{C}$ and $\mathrm{C}^{S}$ are symmetric (recall that $A$ is symmetric provided that $\left.{ }_{A} A_{A} \cong \operatorname{Hom}_{\mathbb{k}}\left({ }_{A} A_{A}, \mathbb{k}\right)\right)$. We refer to [25] for details.

For $w \in W$ with reduced decomposition $w=s_{1} s_{2} \ldots s_{k}$, define the Bott-Samelson C-C-bimodule

$$
\hat{B}_{w}:=\mathrm{C} \otimes_{\mathrm{C}^{s_{1}}} \mathrm{C} \otimes_{\mathrm{C}^{s_{2}}} \mathrm{C} \otimes_{\mathrm{C}^{s_{3}}} \cdots \otimes_{\mathrm{C}^{s_{k}}} \mathrm{C} .
$$

Let $\mathcal{C}$ be a small category equivalent to C-mod. Denote by $\mathscr{S}_{W}=\mathscr{S}_{W, S, V, \mathcal{C}}$ the 2-category which has

- one object $i$ (which should be though of as $\mathcal{C}$ );

- as 1-morphisms, all endofunctors of $\mathcal{C}$ coming from tensoring with bimodules from the additive closure of all Bott-Samelson bimodules;

- as 2-morphisms, all natural transformations of functors.

The 2-category $\mathscr{S}_{W}$ is called the 2-category of Soergel bimodules over C. The nontrivial point here is the fact that it is closed under composition of functors. This is shown in [53]. Moreover, it is also shown in [53] that, for each $w \in W$, the bimodule $\hat{B}_{w}$ contains a unique indecomposable summand $B_{w}$ which does not belong to the additive closure of all $\hat{B}_{x}$, where the length of $x$ is strictly smaller than that of $w$. Bimodules $B_{w}$ are usually called Soergel bimodules (although the name is also used for any direct sum of such bimodules). The 2-category $\mathscr{S}_{W}$ is both finitary and fiat, where $\left(B_{w}, B_{w^{-1}}\right)$ forms an adjoint pair of 1-morphisms, for all $w$.

The theory is inspired by [52] where, for finite Weyl groups, Soergel bimodules appear as "combinatorial description" of indecomposable projective functors on the principal block of the BGG category $\mathcal{O}$ associated with a triangular decomposition of the simple finite dimensional Lie algebra corresponding to $W$, see $[4,5,26]$ for details on the latter. An explicit connection between the 2-category of Soergel bimodules and the Kazhdan-Lusztig basis of the Hecke algebra of $(W, S)$ was established in [16].

\subsection{Singular Soergel bimodules}

Let $(W, S)$ be a finite Coxeter system, $\mathfrak{h}$ a reflection faithful complexified $W$-module and $\mathrm{C}$ the corresponding coinvariant algebra. For each $T \subset S$, let $W^{T}$ be the subgroup of $W$ generated by all $t \in T$ and $C^{T}$ the subalgebra of $W^{T}$-invariants in C. Note that $\mathrm{C}^{\varnothing}=\mathrm{C}$ while $\mathrm{C}^{S}=\mathbb{C}$.

For each $T \subset S$, let $\mathcal{C}^{T}$ be a small category equivalent to $\mathrm{C}^{T}$-mod. Denote by $\mathscr{S} \mathscr{S}_{W}=\mathscr{S} \mathscr{S}_{W, S, C, \mathcal{C}}$ the 2-category which has

- objects $i^{T}$, where $T \subset S$ (each $i^{T}$ should be though of as $\mathcal{C}^{T}$ );

- as 1-morphisms, all endofunctors from $\mathcal{C}^{T}$ to $\mathcal{C}^{R}$ given by

$$
\operatorname{Res}_{\mathrm{C}^{R}}^{\mathrm{C}} \circ \mathrm{F} \circ \mathrm{Ind}_{\mathrm{C}^{T}}^{\mathrm{C}} \text {, }
$$

where $\mathrm{F}$ is given by a usual Soergel bimodule; 
- as 2-morphisms, all natural transformations of functors.

The 2-category $\mathscr{S} \mathscr{S}_{W}$ is called the 2-category of singular Soergel bimodules over C. The 2-category $\mathscr{S} \mathscr{S}_{W}$ is both finitary and fiat.

The 2-category $\mathscr{S} \mathscr{S}_{W}$ also admits an alternative description using projective functors between singular blocks of the BGG category $\mathcal{O}$, see [4,5,52].

\subsection{Finitary quotients of 2-Kac-Moody algebras}

Let $\mathfrak{g}$ be a simple complex finite dimensional Lie algebra and $\dot{U}_{\mathfrak{g}}$ the idempotented version of the universal enveloping algebra of $\mathfrak{g}$, see [35]. The papers $[29,50]$ introduce certain (not finitary) 2-categories whose Grothendieck decategorification is isomorphic to the integral form of $\dot{U}_{\mathfrak{g}}$. In [6] it is further shown that the two (slightly different) constructions in $[29,50]$ give, in fact, biequivalent 2-categories. These are the so-called 2-Kac-Moody algebras of finite type which we will denote $\mathscr{U}_{\mathfrak{g}}$.

Each simple finite dimensional $\mathfrak{g}$-module $V(\lambda)$, where $\lambda$ is the highest weight, admits a categorification in the sense that there exists a 2-representation $\mathbf{M}_{\lambda}$ of $\mathscr{U}_{\mathfrak{g}}$ (even a unique one, up to equivalence, under the additional assumption that the object $\lambda$ is represented by a non-zero semi-simple category) whose Grothendieck decategorification is isomorphic to the integral form of $V(\lambda)$. Let $\mathscr{I}_{\lambda}$ be the kernel of $\mathbf{M}_{\lambda}$. Then $\mathscr{I}_{\lambda}$ is a two-sided 2-ideal of $\mathscr{U}_{\mathfrak{g}}$ and the quotient 2-category $\mathscr{U}_{\mathfrak{g}} / \mathscr{I}_{\lambda}$ is both, finitary and fiat.

\section{Cells and cell 2-representations}

\subsection{Cells}

For a finitary 2-category $\mathscr{C}$, denote by $\mathcal{S}[\mathscr{C}]$ the set of isomorphism classes of indecomposable 1 -objects in $\mathscr{C}$. The set $\mathcal{S}[\mathscr{C}]$ is finite and has the natural multivalued operation $\bullet$ given, for $[\mathrm{F}],[\mathrm{G}] \in \mathcal{S}[\mathscr{C}]$, by

$[F] \bullet[G]=\{[H]: H$ is isomorphic to a summand of $F \circ G\}$.

The operation $\bullet$ is associative (as a multivalued operation) and hence defines on $\mathcal{S}[\mathscr{C}]$ the structure of a multisemigroup, see [41, Section 3] (see also [33] for more details on multisemigroups).

The left partial pre-order $\leq_{L}$ on $\mathcal{S}[\mathscr{C}]$ is defined by setting $[\mathrm{F}] \leq_{L}[\mathrm{G}]$, for $[\mathrm{F}],[\mathrm{G}] \in$ $\mathcal{S}[\mathscr{C}]$, provided that $\mathrm{G}$ is isomorphic to a summand of $\mathrm{H} \circ \mathrm{F}$, for some 1-morphism $\mathrm{H}$. Equivalence classes with respect to $\leq_{L}$ are called left cells and the corresponding equivalence relation is denoted $\sim_{L}$.

The right partial pre-order $\leq_{R}$, the right cells and the corresponding equivalence relation $\sim_{R}$ are defined similarly using multiplication with $\mathrm{H}$ on the right of $\mathrm{F}$. The two-sided partial pre-order $\leq_{J}$, the two-sided cells and the corresponding equivalence relation $\sim_{J}$ are defined similarly using multiplication with $\mathrm{H}_{1}$ and $\mathrm{H}_{2}$ on both sides of $F$. 
These notions are similar and spirit to and generalize the notions of Green's relations and partial orders for semigroups, see [21], and also the notions of Kazhdan-Lusztig cells and order in [27], see also [31]. We refer the reader to [33] for more details and to [31] for a generalization to positively based algebras.

For simplicity, we will say "left cells in $\mathscr{C}$ " instead of "left cells in $\mathcal{S}[\mathscr{C}]$ " and similarly for right and 2-sided cells.

A two-sided cell $\mathcal{J}$ is said to be regular provided that

- any pair of left cells inside $\mathcal{J}$ is incomparable with respect to the left order;

- any pair of right cells inside $\mathcal{J}$ is incomparable with respect to the right order.

A two-sided cell $\mathcal{J}$ is said to be strongly regular provided that it is regular and $|\mathcal{L} \cap \mathcal{R}|=$ 1 , for any left cell $\mathcal{L}$ in $\mathcal{J}$ and any right cell $\mathcal{R}$ in $\mathcal{J}$. We refer to [40, Subsection 4.8] for details. A two-sided cell $\mathcal{J}$ is said to be idempotent provided that it contains three elements $\mathrm{F}, \mathrm{G}$ and $\mathrm{H}$ (not necessarily distinct) such that $\mathrm{F}$ is isomorphic to a direct summand of $\mathrm{G} \circ \mathrm{H}$, see [10, Subsection 2.3]. The following is [31, Corollary 19].

Proposition 1 Each idempotent two-sided cell of finitary 2-category is regular, in particular, each two-sided cell of (weakly) fiat 2-category is regular.

\subsection{Cell 2-representations}

Let $\mathscr{C}$ be a finitary 2 -category and $\mathcal{L}$ a left cell in $\mathscr{C}$. Then there is an object $i=i_{\mathcal{L}} \in \mathscr{C}$ such that all 1-morphisms in $\mathcal{L}$ start from $i$. Consider the principal 2-representation $\mathbf{P}_{\text {i. }}$

For each $j \in \mathscr{C}$, denote by $\mathbf{M}(j)$ the additive closure in $\mathbf{P}_{i}(j)$ of all indecomposable 1-morphisms $F \in \mathbf{P}_{i}(j)=\mathscr{C}(i, j)$ satisfying $\mathcal{L} \leq_{L} F$ (note that the latter notation makes sense as $\mathcal{L}$ is a left cell). Then $\mathbf{M}$ has the natural structure of a 2-representation of $\mathscr{C}$ which is inherited from $\mathbf{P}_{i}$ by restriction. The following lemma can be found e.g. in [44, Lemma 3].

Lemma 2 The 2-representation $\boldsymbol{M}$ has a unique maximal $\mathscr{C}$-invariant ideal $\mathbf{I}$.

The quotient 2-representation $\mathbf{M} / \mathbf{I}$ is called the cell 2-representation corresponding to $\mathcal{L}$ and denoted $\mathbf{C}_{\mathcal{L}}$. The construction presented here first appears in [41, Subsection 6.5]. It follows directly from the construction that isomorphism classes of indecomposable objects in

$$
\coprod_{j \in \mathscr{C}} \mathbf{C}_{\mathcal{L}}(j)
$$

correspond bijectively to elements in $\mathcal{L}$. Note that two cell 2-representations $\mathbf{C}_{\mathcal{L}}$ and $\mathbf{C}_{\mathcal{L}^{\prime}}$ might be equivalent even in the case $\mathcal{L} \neq \mathcal{L}^{\prime}$. The following is [45, Theorem 3.1].

Theorem 3 Let $\mathscr{C}$ be a weakly fiat 2-category in which all two-sided cells are strongly regular. Then, for any left cells $\mathcal{L}$ and $\mathcal{L}^{\prime}$ in $\mathscr{C}$, we have $\boldsymbol{C}_{\mathcal{L}} \cong \boldsymbol{C}_{\mathcal{L}^{\prime}}$ if and only if $\mathcal{L}$ and $\mathcal{L}^{\prime}$ belong to the same two-sided cell in $\mathscr{C}$.

Cell 2-representations can be viewed as natural 2-analogues of semigroups representations associated to left cells, see [20, Subsection 11.2]. 


\subsection{Basic properties of cell 2-representations}

Let $\mathscr{C}$ be a finitary 2-category and $\mathbf{M} \in \mathscr{C}$-afmod. We will say that $\mathbf{M}$ is transitive provided that, for any $i, j \in \mathscr{C}$ and for any indecomposable $X \in \mathbf{M}(i)$ and $Y \in \mathbf{M}(j)$, there is a 1-morphism $\mathrm{F} \in \mathscr{C}(i, j)$ such that $Y$ is isomorphic to a summand of $\mathrm{F} X$.

Directly from the definition of left cells and the construction of cell 2-representations it follows that each cell 2-representation is transitive.

We will say that $\mathbf{M}$ is simple provided that $\mathbf{M}$ has no proper $\mathscr{C}$-stable ideals. Note that every simple 2-representation is automatically transitive.

Directly from construction of cell 2-representations and Lemma 2 it follows that each cell 2-representation is simple. So, we have the following claim (see [44, Section 3]):

Proposition 4 Every cell 2-representation of a finitary 2-category is both simple and transitive.

\subsection{Alternative construction of cell 2-representations for fiat 2-categories}

Let $\mathscr{C}$ be a fiat 2-category, $\mathcal{L}$ a left cell in $\mathscr{C}$ and $i=i_{\mathcal{L}}$. Consider the principal 2-representation $\mathbf{P}_{i}$ and its abelianization $\overline{\mathbf{P}}_{i}$. For $j \in \mathscr{C}$, projective objects in $\overline{\mathbf{P}}_{i}(j)$ correspond (up to isomorphism) to 1-morphisms $\mathrm{F} \in \mathscr{C}(i, j)$ and are denoted $P_{\mathrm{F}}$. The simple top of $P_{\mathrm{F}}$ is denoted $L_{\mathrm{F}}$.

In the fiat case, [40, Section 4] provides an alternative construction of cell 2representation which is based on the notion of Duflo involution. The following is [40, Proposition 17]:

Proposition 5 The left cell $\mathcal{L}$ contains a unique element $G=G_{\mathcal{L}}$, called the Duflo involution in $\mathcal{L}$, such that there is a sub-object $K$ of the projective object $P_{\mathbb{1}_{i}}$ satisfying the following conditions:

(a) $F\left(P_{1_{i}} / K\right)=0$, for all $F \in \mathcal{L}$;

(b) $F \operatorname{top}(K) \neq 0$, for all $F \in \mathcal{L}$;

(c) $\operatorname{top}(K) \cong L_{G}$.

For $j \in \mathscr{C}$, denote by $\mathbf{N}(j)$ the additive closure, in $\overline{\mathbf{P}}_{i}(j)$, of all elements of the form $F L_{G}$, where $F \in \mathcal{L} \cap \mathscr{C}(i, j)$. The following is [41, Proposition 22]:

Proposition 6 (i) The assignment $\boldsymbol{N}$ inherits, by restriction from $\overline{\boldsymbol{P}}_{i}$, the natural structure of a 2-representation of $\mathscr{C}$.

(ii) The 2-representations $\boldsymbol{N}$ and $\boldsymbol{C}_{\mathcal{L}}$ are equivalent.

The notion of Duflo involution was generalized to some non-fiat 2-categories in [57]. In [56, Example 8] (see also [32, Subsection 9.3]) one finds an example of a fiat 2 -category with a left cell $\mathcal{L}$ such that the corresponding Duflo involution $\mathrm{G}$ satisfies $\mathrm{G} \not \mathrm{G}^{\star}$. 


\section{Simple transitive 2-representations}

\subsection{Weak Jordan-Hölder theory}

In this subsection we overview the weak Jordan-Hölder theory for additive 2representations of finitary 2-categories developed in [44, Section 4]. Here simple transitive 2-representations play a crucial role. We start with the following observation which is just a variation of Lemma 2, see [44, Lemma 4].

Lemma 7 Every transitive 2-representation of a finitary 2-category has a unique simple transitive quotient.

Let $\mathscr{C}$ be a finitary 2-category and $\mathbf{M} \in \mathscr{C}$-afmod. Let $\operatorname{Ind}(\mathbf{M})$ be the (finite!) set of isomorphism classes of indecomposable objects in

$$
\coprod_{i \in \mathscr{C}} \mathbf{M}(i) .
$$

The action pre-order $\rightarrow \mathscr{C}$ on Ind(M) is defined as follows: $X \rightarrow_{\mathscr{C}} Y$ provided that $Y$ is isomorphic to a direct summand of $\mathrm{F} X$, for some 1-morphism $\mathrm{F}$ in $\mathscr{C}$. Consider a filtration

$$
\varnothing=\mathcal{Q}_{0} \subsetneq \mathcal{Q}_{1} \subsetneq \cdots \subsetneq \mathcal{Q}_{m}=\operatorname{Ind}(\mathbf{M})
$$

such that, for each $i=1,2, \ldots, m$,

- the set $\mathcal{Q}_{i} \backslash \mathcal{Q}_{i-1}$ is an equivalence class with respect to $\rightarrow \mathscr{C}$,

- the set $\mathcal{Q}_{i}$ has the property that, for all $X \in \mathcal{Q}_{i}$ and $Y \in \operatorname{Ind}(\mathbf{M})$ such that $X \rightarrow \mathscr{C} Y$, we have $Y \in \mathcal{Q}_{i}$.

For $i=1,2, \ldots, m$ and $j \in \mathscr{C}$, let $\mathbf{M}_{i}(j)$ denote the additive closure in $\mathbf{M}(j)$ of all objects from $\mathcal{Q}_{i} \cap \mathbf{M}(j)$. Furthermore, we denote by $\mathbf{I}_{i}(j)$ the ideal of $\mathbf{M}_{i}(j)$ generated by all objects from $\mathcal{Q}_{i-1} \cap \mathbf{M}(j)$.

The assignment $\mathbf{M}_{i}$ inherits, by restriction from $\mathbf{M}$, the structure of a 2representation of $\mathscr{C}$, moreover, $\mathbf{I}_{i}$ is a $\mathscr{C}$-stable ideal in $\mathbf{M}_{i}$. Hence we have a filtration

$$
0 \subset \mathbf{M}_{1} \subset \mathbf{M}_{2} \subset \cdots \subset \mathbf{M}_{m}=\mathbf{M}
$$

of $\mathbf{M}$ by 2-representations. This is a weak Jordan-Hölder series of $\mathbf{M}$. By construction, the 2-representation $\mathbf{M}_{i} / \mathbf{I}_{i}$ is transitive and we can denote by $\mathbf{L}_{i}$ its unique simple transitive quotient, given by Lemma 7 . For a 2 -representation $\mathbf{N}$, denote by $[\mathbf{N}]$ the equivalence class of $\mathbf{N}$. The multiset $\left\{\left[\mathbf{L}_{i}\right]: i=1,2, \ldots, m\right\}$ is then the multiset of weak Jordan-Hölder subquotients of $\mathbf{M}$. The following is [44, Theorem 8].

Theorem 8 The multiset $\left\{\left[\boldsymbol{L}_{i}\right]: i=1,2, \ldots, m\right\}$ does not depend on the choice of the filtration (3). 


\subsection{How many simple transitive 2-representations do we have?}

Theorem 8 motivates the study of simple transitive 2-representations for finitary 2categories. Note that simple 2-representations are automatically transitive and hence the name is slightly redundant. However, the name simple transitive has an advantage that it addresses both layers of the representation structure:

- transitivity refers to the discrete layer of objects;

- simplicity refers to the $\mathbb{k}$-linear layer of morphisms.

For an arbitrary finitary 2 -category $\mathscr{C}$, we thus have a general problem:

Problem 9 Classify all simple transitive 2-representations of $\mathscr{C}$, up to equivalence.

Later on we will survey the cases in which this problem is solved, however, for general $\mathscr{C}$, even for general fiat $\mathscr{C}$, it is wide open.

Of course, one could try to draw parallels with finite dimensional algebras. One very easy fact from the classical representation theory is that every finite dimensional $\mathbb{k}$-algebra has only a finite number of isomorphism classes of simple modules. The corresponding statement in 2-representation theory is still open. At the moment this seems to be one of the major challenges in this theory.

Question 10 Is it true that, for any finitary (fiat) 2-category $\mathscr{C}$, the number of equivalence classes of simple transitive 2-representations of $\mathscr{C}$ is finite?

In all cases in which the answer to Problem 9 is known (see below), the number of of equivalence classes of simple transitive 2-representations is indeed finite.

\subsection{Finitary 2-categories of type I}

A finitary 2-category $\mathscr{C}$ is said to be of type I provided that every simple transitive 2-representation of $\mathscr{C}$ is equivalent to a cell 2-representations. Thus, for a finitary 2-category of type I, Problem 9 has a fairly easy answer (modulo comparison of cell 2-representations with each other). Moreover, for a finitary 2-category of type I, Question 10 has positive answer. The first example of finitary 2-categories of type I is provided by [44, Theorem 18] and [45, Theorem 33].

Theorem 11 Every weakly fiat 2-category with strongly regular two-sided cells is of type $I$.

Here we also note that many results in [40]-[44] assume that a certain numerical condition is satisfied. This assumption was rendered superfluous by [45, Proposition 1].

As a special case of Theorem 11 , we have that, the 2-category $\mathscr{C}_{A}$, for a selfinjective finite dimensional $\mathbb{k}$-algebra $A$, see Sect. 3.2, is of type I. Another special case of Theorem 11 is that the 2-category $\mathscr{S}_{W}$ of Soergel bimodules in Weyl type $A$ (that is when $W$ is isomorphic to the symmetric group), see Sect. 3.5, is of type I. Furthermore, all finitary quotients of 2-Kac-Moody algebras from Sect. 3.7 are of type I, see [44, Subsection 7.2] for details. 
Simple transitive 2-representations of the 2-category $\mathscr{C}_{A}$ were also studied for some $A$ which are not self-injective (in this case $\mathscr{C}_{A}$ is not weakly fiat). The first result in this direction was the following statement, which is the main result of [47].

Theorem 12 For $A=A_{2}$ or $A_{3}$ as in Sect. 3.3, the corresponding 2-category $\mathscr{C}_{A}$ is of type $I$.

Despite of the fact that $A$ as in Theorem 12 is not self-injective, it has a non-zero projective-injective module. The latter plays a crucial role in the arguments. Recently, based on some progress made in [31] and [32], Theorem 12 was generalized in [48] as follows:

Theorem 13 Let A be a basic connected $\mathbb{k}$-algebra which has a non-zero projectiveinjective module and which is directed in the sense that the Gabriel quiver of the algebra A has neither loops nor oriented cycles. Then the corresponding 2-category $\mathscr{C}_{A}$ is of type $I$.

When $A$ does not have any non-zero projective-injective module, the approach of $[44,47,48]$ fails. Only one special case (the smallest one) was recently completed in [46, Theorem 6] (partially based on [44, Subsection 7.1]).

Theorem 14 For $A=\mathbb{k}[x, y] /\left(x^{2}, y^{2}, x y\right)$, the corresponding 2-category $\mathscr{C}_{A}$ is of type I.

After all the cases listed above, the following question is rather natural:

Question 15 Is it true that the 2-category $\mathscr{C}_{A}$ is of type I, for any A?

Apart from the cases listed above, there is a number of other type I examples. The following result in [59, Theorem 6.1].

Theorem 16 The 2-category of Soergel bimodules in Weyl type $B_{2}$ is of type I.

One interesting difference of the latter case compared to all other cases listed above is the fact that, for the 2-category of Soergel bimodules in Weyl type $B_{2}$, there are non-equivalent cell 2-representations which correspond to left cells inside the same two-sided cell.

Let $\left(W, S\right.$ ) be a finite Coxeter system. The corresponding 2-category $\mathscr{S}_{W}$ of Soergel bimodules, see Sect. 3.5, has a unique minimum two-sided cell consisting of the identity 1-morphism. If we take this minimum two-sided cell away, in what remains there is again a unique minimum two-sided cell $\mathcal{J}$. This two-sided cell contains, in particular, all Soergel bimodules of the form $\mathrm{C} \otimes_{\mathrm{C}^{s}} \mathrm{C}$, where $s \in S$. There is a unique 2-ideal $\mathscr{I}$ in $\mathscr{S}_{W}$ which is maximal, with respect to inclusions, in the set of all 2-ideals in $\mathscr{S}_{W}$ that do not contain identity 2-morphisms for 1-morphisms in $\mathcal{J}$. The quotient $\mathscr{S}_{W}:=\mathscr{S}_{W} / \mathscr{J}$ is called the small quotient of $\mathscr{S}_{W}$, see [32, Subsection 3.2]. The 2-category $\mathscr{S}_{W}$ inherits from $\mathscr{S}_{W}$ the structure of a fiat 2-category. The following result can be found in [32, Sections 6, 7 and 8].

Theorem 17 (i) If $|S|>2$, then $\mathscr{S}_{W}$ is of type $I$.

(ii) If $(W, S)$ is of Coxeter type $I_{2} \overline{(n)}$, with $n>4$, then $\mathscr{S}_{W}$ is of type I if and only if $n$ is odd. 


\subsection{Finitary 2-categories that are not of type I}

First, rather degenerate examples of finitary 2-categories which are not of type I were constructed already in [44, Subsection 3.2]. They are inspired by transitive group actions. Each finite group structure can be extended to a fiat 2-category in a fairly obvious way (by adding formal direct sums of elements and linearizing spaces of identity 2-morphisms). The resulting 2-category has just one left cell and the corresponding cell 2-representation is, morally, the left regular representation of the group. However, simple transitive 2-representations correspond to transitive actions of the original group on sets. The latter are given by action on (left) cosets modulo subgroups. In particular, we get a lot of simple transitive 2-representations which are not cell 2-representations. The example, however, feels rather artificial.

The first more "natural" example was constructed in [37]. Let $(W, S)$ be a Coxeter system of type $I_{2}(n)$, with $n>3$. Consider the small quotient $\mathscr{S}_{W}$ of the 2-category of Soergel bimodules. This is a fiat 2-category with two two-sided cells. The minimum one consists just of the identity 1-morphisms. The maximum one is not strongly regular. Let $S=\{s, t\}$. Denote by $\mathscr{Q}_{n}$ the 2 -full sub-2-category of $\mathscr{S}_{W}$ given by all 1morphisms in the additive closure of the identity 1-morphisms and of all 1-morphisms $\mathrm{F}$ which lie in the same right cell and in the same left cell as the 1-morphisms given by $\mathrm{C} \otimes_{\mathrm{C}^{s}} \mathrm{C}$. The main result of [37] is the following:

\section{Theorem 18 (i) The 2-category $\mathscr{Q}_{5}$ is of type I.}

(ii) The 2-category $\mathscr{Q}_{4}$ is not of type I. In fact, $\mathscr{Q}_{4}$ has a unique (up to equivalence) simple transitive 2-representation which is not equivalent to any of two cell 2-representations.

The major part of [37] is devoted to an explicit construction of this additional simple transitive 2-representation of $\mathscr{Q}_{4}$. The construction is very technical and is based on the following idea: One of the two cell 2-representations of $\mathscr{Q}_{4}$ has an invertible automorphism which swaps the isomorphism classes of the two non-isomorphic indecomposable objects in the underlying category of this 2-representation. The additional simple transitive 2-representation is constructed using the orbit category with respect to this non-trivial automorphism. The main issue is that this automorphism is not strict (as homomorphisms between 2-representations are not strict) and so it requires a lot of technical effort to go around this complication. Based on this construction, the following statement was proved in [32, Sections 7].

Theorem 19 Let $(W, S)$ be of Coxeter type $I_{2}(n)$, with $n>4$ even. Then $\mathscr{S}_{W}$ is not of type I. Moreover, the following holds:

(i) Two of the three cell 2-representations of $\mathscr{S}_{W}$ have an invertible automorphism which is not isomorphic to the identity. The orbit construction as in [37] with respect to this automorphism produces a new simple transitive 2-representation of $\mathscr{S}_{W}$.

(ii) If $n \neq 12,18,30$, then every simple transitive 2-representation of $\mathscr{S}_{W}$ is equivalent to either a cell 2-representation or one of the 2-representations constructed in (i). 


\subsection{Schur's lemma}

As we saw in the previous subsection, endomorphisms of cell 2-representations play an important role in this study. This naturally raises the following problem:

Problem 20 Describe the (properties of the) bicategory of endomorphisms of a simple transitive 2-representation of a finitary 2-category.

The only known result in this direction is the following statement which is [42, Theorem 16].

Theorem 21 Let $\mathscr{C}$ be a fiat 2-category, $\mathcal{J}$ a strongly regular two-sided cell in $\mathscr{C}$ and $\mathcal{L}$ a left cell in $\mathcal{J}$. Then any endomorphism of the cell 2-representation $\boldsymbol{C}_{\mathcal{L}}$ is isomorphic to the direct sum of a number of copies of the identity endomorphism $I D_{C_{\mathcal{L}}}$. Moreover, the endomorphism space (given by all modifications) of $I D_{C_{\mathcal{L}}}$ consist just of scalar multiplies of the identity modification.

\subsection{Apex}

The following is [10, Lemma 1].

Lemma 22 Let $\mathscr{C}$ be a finitary 2-category and $\boldsymbol{M}$ a transitive 2-representation of $\mathscr{C}$. There is a unique two sided cell $\mathcal{J}=\mathcal{J}_{M}$ which is maximal, with respect to the twosided order, in the set of all two-sided cells that contain 1-morphisms which are not annihilated by $\boldsymbol{M}$. The two-sided cell $\mathcal{J}$ is idempotent.

The two-sided cell $\mathcal{J}$ is called the apex of $\mathbf{M}$. The general problem of classification of all simple transitive 2-representations of $\mathscr{C}$ thus splits naturally into subproblems to classify simple transitive 2-representations of $\mathscr{C}$ with a fixed apex $\mathcal{J}$ (which should be an idempotent two-sided cell). Quite often, this simplifies the problem, due to the following result which is proved analogously to [44, Theorem 18].

Theorem 23 Let $\mathscr{C}$ be a weakly fiat 2-category, $\mathcal{J}$ a strongly regular two-sided cell in $\mathscr{C}$ and $\boldsymbol{M}$ a simple transitive 2-representation of $\mathscr{C}$ with apex $\mathcal{J}$. Then $\boldsymbol{M}$ is equivalent to a cell 2-representation.

\subsection{Connection to integral matrices}

Let $\mathscr{C}$ be a finitary 2-category and $\mathbf{M} \in \mathscr{C}$-afmod. Let Ind(M) be as in Sect. 5.1. Then, to any 1-morphism $\mathrm{F}$ in $\mathscr{C}$, one can associate a matrix $[\mathrm{F}]_{\mathbf{M}}$ whose rows and columns are indexed by elements in $\operatorname{Ind}(\mathbf{M})$ and the intersection of the $X$-row and $Y$-column gives the multiplicity of $X$ as a summand of $\mathrm{F} Y$. The following observation is [44, Lemma 11(ii)].

Lemma 24 Assume that $\boldsymbol{M}$ is transitive and that $F$ contains, as summands, representatives from all isomorphism classes of indecomposable 1-morphisms in $\mathscr{C}$. Then all coefficients in $[F]_{M}$ are positive. 
This observation allows one to use the classical Perron-Frobenius Theorem (see $[18,19,49])$ in the study of simple transitive 2-representations. This is an important ingredient in the arguments in $[44,46,47,59]$.

The above observation also provides some evidence for the general positive answer to Question 10. Indeed, the Grothendieck decategorification of a finitary 2-category $\mathscr{C}$ gives a finite dimensional $\mathbb{k}$-algebra, call it $A$. For each 1 -morphism $\mathrm{F}$ in $\mathscr{C}$, we thus have the minimal polynomial $g_{\mathrm{F}}(\lambda)$ for the class $[\mathrm{F}]_{\oplus}$ in $A$. Now, if $\mathbf{M} \in \mathscr{C}$-afmod, then $[\mathbf{M}]_{\oplus}$ gives rise to an $A$-module and hence

$$
g_{\mathrm{F}}\left([\mathrm{F}]_{\mathbf{M}}\right)=0 .
$$

Therefore, if $\mathbf{M}$ is transitive and $\mathbf{F}$ contains, as summands, representatives from all isomorphism classes of indecomposable 1-morphisms in $\mathscr{C}$, then, because of Lemma 24 and [60, Theorem 3.2], there are only finitely many possibilities for the matrix $[\mathrm{F}]_{\mathbf{M}}$. Consequently, there are only finitely many possibilities for matrices $[\mathrm{G}]_{\mathbf{M}}$, where $\mathrm{G}$ is an indecomposable 1-morphism in $\mathscr{C}$.

In many papers, for instance, in $[44,45,47,48,59]$, the classification problem was approached in two steps. The first step addressed classification of all possibilities for matrices $[\mathrm{F}]_{\mathbf{M}}$. The second step studied actual 2-representations for each solution provided by the first step. In case of the 2-category $\mathscr{C}_{A}$, the first step studied matrices $M$ with positive integer coefficients satisfying $M^{2}=\operatorname{dim}(A) M$. This is an interesting combinatorial problem which was investigated in detail in [60].

\subsection{Approach using (co)algebra objects}

The story with the cases $n=12,18,30$ in Theorem 19(ii) was quite interesting. The detailed study of integral matrices, as outlined in Sect. 5.7, suggested in these cases possibility of existence of simple transitive 2-representations of $\mathscr{S}_{W}$ which are neither cell 2-representations nor the ones constructed in Theorem 19(i). These 2representations were constructed in [39], based on [15], using diagrammatic calculus. Under the additional assumption of gradeability, it was shown that, together with cell 2-representations and the 2-representations constructed in Theorem 19(i), these exhaust all simple transitive 2-representations of $\mathscr{S}_{W}$.

Unfortunately, the diagrammatic calculus is not really compatible with our definitions of 2-categories. This raised a natural problem to reformulate the results of [39] in some language compatible with our definitions. This was achieved in [38] using ideas of [14] related to the study of algebra and coalgebra objects in 2-categories. More precisely, the following is [38, Theorem 9].

Theorem 25 Let $\mathscr{C}$ be a fiat 2-category and $M$ a transitive 2-representations of $\mathscr{C}$. Then there is a coalgebra object $A$ in the injective abelianization $\mathscr{C}$ of $\mathscr{C}$ such that $\boldsymbol{M}$ is equivalent to the 2-representation of $\mathscr{C}$ given by the action of $\mathscr{C}$ on the category of injective right $A$-comodule objects in $\mathscr{C}$.

For cell 2-representations, the corresponding coalgebra objects turn out to be related to Duflo involutions, see [38, Subsection 6.3]. Theorem 25 motivates the following general problem: 
Problem 26 Classify all coalgebra objects in $\underline{\mathscr{C}}$, up to isomorphism.

In this general formulation, Problem 26 is certainly more difficult than Problem 9. However, the very useful side of Theorem 25 is that one can construct simple transitive 2-representations by guessing the corresponding coalgebra objects (as it was done for the "exotic" simple transitive 2-representations of $\mathscr{S}_{W}$ in types $I_{2}(12), I_{2}(18)$ and $I_{2}(30)$ in [38]).

\section{Other classes of 2-representations and related questions}

\subsection{Isotypic 2-representations}

Let $\mathscr{C}$ be a finitary 2-category and $\mathbf{M}$ a 2-representation of $\mathscr{C}$. We will say that $\mathbf{M}$ is isotypic provided that all weak Jordan-Hölder subquotients of $\mathbf{M}$ are equivalent, see [51, Subsection 4.3.4] and [45, Subsection 3.6].

For any 2-representation $\mathbf{M}$ of $\mathscr{C}$ and any finitary $\mathbb{k}$-linear 2-category $\mathcal{A}$ one defines the inflation $\mathbf{M}^{\bigotimes \mathcal{A}}$ of $\mathbf{M}$ by $\mathcal{A}$ as the 2-representation of $\mathscr{C}$ which sends each $i \in \mathscr{C}$ to the tensor product $\mathbf{M}(i) \otimes \mathcal{A}$ and defines the action of $\mathscr{C}$ on the objects and morphisms in these tensor products by acting on the first component, see [45, Subsection 3.6] for details. The following result is [45, Theorem 4].

Theorem 27 Let $\mathscr{C}$ be a weakly fiat 2-category with a unique maximal two-sided cell $\mathcal{J}$. Let $\mathcal{L}$ be a left cell in $\mathcal{J}$. Assume that $\mathcal{J}$ is strongly regular and that any non-zero 2-ideal of $\mathscr{C}$ contains the identity 2-morphism, for some 1-morphism in $\mathcal{J}$. Then any isotypic faithful 2-representation of $\mathscr{C}$ is equivalent to an inflation of the cell 2-representation $\boldsymbol{C}_{\mathcal{L}}$.

For finitary quotients of 2-Kac-Moody algebras, the statement of Theorem 28 can be deduced from [51, Subsection 4.3.4]. Compared to the general case, the case of 2-KacMoody algebras is substantially simplified by existence of idempotent 1-morphisms in each two-sided cell. A challenging problem related to isotypic 2-representation is the following:

Problem 28 Classify faithful isotypic 2-representation for an arbitrary weakly fiat 2 -category $\mathscr{C}$ with unique maximal two-sided cell $\mathcal{J}$ under the assumption that that $\mathcal{J}$ is strongly regular.

In the easiest case, this problem will appear in the next subsection.

\subsection{All 2-representations}

The question of classification of all 2-representations, for a given finitary 2-category $\mathscr{C}$, is open in all non-trivial case. The only trivial case is the case when the only indecomposable 1-morphisms in $\mathscr{C}$ are the identities, up to isomorphism. It is certainly enough to consider the case when $\mathscr{C}$ has one object, say $i$. Up to biequivalence, we may also assume that $\nVdash_{i}$ is the only indecomposable 1 -morphism in $\mathscr{C}$ (on the nose 
and not just up to isomorphism). Then, directly from the definition, we have that a 2-representation of such $\mathscr{C}$ is given by a pair $(Q, \varphi)$, where $Q$ is a finite dimensional $\mathbb{k}$-algebra and $\varphi$ is an algebra homomorphism from End $\left(\nVdash_{i}\right)$ to the center of $Q$. Then $\mathscr{C}$ acts on a small category equivalent to $Q$-proj in the obvious way. Note that all 2-representations of $\mathscr{C}$ are isotypic. Furthermore, $\mathscr{C}$ satisfies the assumptions of Theorem 28 if and only if $\operatorname{End}\left(\nVdash_{i}\right) \cong \mathbb{k}$.

The first non-trivial case to consider would be the following:

Problem 29 Classify, up to equivalence, all finitary additive 2-representations of $\mathscr{C}_{D}$, where $D=\mathbb{C}[x] /\left(x^{2}\right)$.

\subsection{Discrete extensions between 2-representations}

A major challenge in 2-representation theory is the following:

Problem 30 Develop a sensible homological theory for the study of 2-representations.

A fairly naive attempt to define some analogue of $\mathrm{Ext}^{1}$ for 2-representations was made in [10].

Let $\mathscr{C}$ be a finitary 2-category and $\mathbf{M} \in \mathscr{C}$-afmod. For each $i \in \mathscr{C}$, choose a full, additive, idempotents split and isomorphism closed subcategory $\mathbf{K}(i)$ of $\mathbf{M}(i)$ such that $\mathbf{K}$ becomes a sub-2-representation of $\mathscr{C}$ by restriction. Let $\mathbf{I}$ be the ideal of $\mathbf{M}$ generated by $\mathbf{K}$ and $\mathbf{N}:=\mathbf{M} / \mathbf{I}$. Then the sequence

$$
0 \rightarrow \mathbf{K} \stackrel{\Phi}{\longrightarrow} \mathbf{M} \stackrel{\Psi}{\longrightarrow} \mathbf{N} \rightarrow 0
$$

where $\Phi$ is the natural inclusion and $\Psi$ is the natural projection, will be called a short exact sequence of 2 -representations of $\mathscr{C}$. The discrete extension $\Theta$ realized by (4) is the subset of $\mathcal{S}[\mathscr{C}]$ that consists of all classes [F] for which there exist an indecomposable object $X$ in some $\mathbf{M}(i) \backslash \mathbf{K}(i)$ and an indecomposable object $Y$ in some $\mathbf{K}(j)$ such that $Y$ is isomorphic to a summand of $F X$.

For $\mathbf{K}^{\prime}, \mathbf{N}^{\prime} \in \mathscr{C}$-afmod, the set $\operatorname{Dext}\left(\mathbf{N}^{\prime}, \mathbf{K}^{\prime}\right)$ of discrete extensions from $\mathbf{N}^{\prime}$ to $\mathbf{K}^{\prime}$ consists of all possible $\Theta$ which are realized by some short exact sequence (4) with $\mathbf{K}$ equivalent to $\mathbf{K}^{\prime}$ and $\mathbf{N}$ equivalent to $\mathbf{N}^{\prime}$.

In many case, a very useful piece of information is to know whether $\operatorname{Dext}(\mathbf{N}, \mathbf{K})$ is empty (i.e. the first discrete extension vanishes) or not. In a number of cases, one could also either explicitly describe all elements in $\operatorname{Dext}(\mathbf{N}, \mathbf{K})$ or at least give a reasonable estimate of how they look like. Vanishing of discrete extensions between certain simple transitive 2-representations appears in a disguised form and is an essential part of the arguments in $[44,45]$. The following is [10, Theorem 25].

Theorem 31 Let $\mathscr{C}$ be a weakly fiat 2-category, $\boldsymbol{K}$ a transitive 2-representation of $\mathscr{C}$ with apex $\mathcal{J}_{K}$, and $N$ a transitive 2-representation of $\mathscr{C}$ with apex $\mathcal{J}_{N}$. Assume that, for any left cell $\mathcal{L}$ in $\mathcal{J}_{N}$, there exists a left cell $\mathcal{L}^{\prime}$ in $\mathcal{J}_{\boldsymbol{K}}$ such that $\mathcal{L} \geq_{L} \mathcal{L}^{\prime}$. Then $\operatorname{Dext}(\boldsymbol{N}, \boldsymbol{K})=\varnothing$. 
As a consequence of Theorem 31, all discrete self-extensions for transitive 2-representations of weakly fiat 2-categories vanish.

The results in [10, Subsection 7.2] suggest that the answer to Question 32 might be interesting and is not obvious.

Question 32 What is $\operatorname{Dext}(\boldsymbol{N}, \boldsymbol{K})$, for any pair $(\boldsymbol{N}, \boldsymbol{K})$ of simple transitive 2representations of the 2-category $\mathscr{S}_{W}$ of Soergel bimodules in Weyl type A?

\subsection{Applications}

The first, rather spectacular, application of classification of certain classes of 2representations appears in [11]. More precisely, [11, Proposition 5.26] classifies, up to equivalence, all 2-representations of the 2-Kac-Moody version of $\mathfrak{s l}_{2}$ which satisfy a number of natural assumptions. This is an essential ingredient in the proof of derived equivalence for certain blocks of the symmetric group, see [11, Theorem 7.6]. Similar ideology was used, in particular, to describe blocks of Lie superalgebras, see, for example, $[7,8]$ and references therein.

In [30], classification of simple transitive 2-representation for the 2-category of Soergel bimodules in type $A$ (cf. Theorem 11) was used to classify indecomposable projective functors on the principal block of BGG category $\mathcal{O}$ for $\mathfrak{s l}_{n}$.

Acknowledgements The author is partially supported by the Swedish Research Council and Göran Gustafsson Stiftelse. The authors thanks the organizers of the Brazilian Algebra Meeting for invitation to give the series of lecture on higher representation theory.

Open Access This article is distributed under the terms of the Creative Commons Attribution 4.0 International License (http://creativecommons.org/licenses/by/4.0/), which permits unrestricted use, distribution, and reproduction in any medium, provided you give appropriate credit to the original author(s) and the source, provide a link to the Creative Commons license, and indicate if changes were made.

\section{References}

1. Bass, H.: Algebraic K-Theory, xx+762 pp. W. A. Benjamin, Inc., New York (1968)

2. Beliakova, A., Guliyev, Z., Habiro, K., Lauda, A.: Trace as an alternative decategorification functor. Acta Math. Vietnam. 39(4), 425-480 (2014)

3. Bernstein, J., Frenkel, I., Khovanov, M.: A categorification of the Temperley-Lieb algebra and Schur quotients of $U\left(\mathfrak{s l}_{2}\right)$ via projective and Zuckerman functors. Sel. Math. 5(2), 199-241 (1999)

4. Bernstenn, I., Gelfand, I., Gelfand, S.: A certain category of $\mathfrak{g}$-modules. Funkcional. Anal. i Prilozen. 10(2), 1-8 (1976)

5. Bernstenn, I., Gelfand, S.: Tensor products of finite- and infinite-dimensional representations of semisimple Lie algebras. Compos. Math. 41(2), 245-285 (1980)

6. Brundan, J.: On the definition of Kac-Moody 2-category. Math. Ann. 364(1-2), 353-372 (2016)

7. Brundan, J., Losev, I., Webster, B.: Tensor product categorifications and the super Kazhdan-Lusztig conjecture. Preprint. arXiv:1310.0349. To appear in IMRN

8. Brundan, J., Stroppel, C.: Highest weight categories arising from Khovanov's diagram algebra IV: the general linear supergroup. J. Eur. Math. Soc. 14(2), 373-419 (2012)

9. Butler, M.C.R., Ringel, C.M.: Auslander-Reiten sequences with few middle terms and applications to string algebras. Commun. Algebra 15(1-2), 145-179 (1987)

10. Chan, A., Mazorchuk, V.: Diagrams and discrete extensions for finitary 2-representations. Preprint. arXiv: 1601.00080 
11. Chuang, J., Rouquier, R.: Derived equivalences for symmetric groups and $\mathfrak{s l}_{2}$-categorification. Ann. Math. 167(1), 245-298 (2008)

12. Crane, L.: Clock and category: is quantum gravity algebraic? J. Math. Phys. 36(11), 6180-6193 (1995)

13. Crane, L., Frenkel, I.: Four-dimensional topological quantum field theory, Hopf categories, and the canonical bases. Topol. Phys. J. Math. Phys. 35(10), 5136-5154 (1994)

14. Etingof, P., Gelaki, S., Nikshych, D., Ostrik, V.: Tensor categories. Mathematical Surveys and Monographs, Vol. 205. American Mathematical Society, Providence, RI (2015)

15. Elias, B.: The two-color Soergel calculus. Compos. Math. 152(2), 327-398 (2016)

16. Elias, B., Williamson, G.: The Hodge theory of Soergel bimodules. Ann. Math. 180(3), 1089-1136 (2014)

17. Freyd, P.: Representations in abelian categories. In: Proceedings of the Conference on Categorical Algebra, pp. 95-120. Springer, New York (1966)

18. Frobenius, G.: Über Matrizen aus positiven Elementen, 1. Sitzungsber. Königl. Preuss. Akad. Wiss. (1908), 471-476

19. Frobenius, G.: Über Matrizen aus positiven Elementen, 2. Sitzungsber. Königl. Preuss. Akad. Wiss., 514-518, (1909)

20. Ganyushkin, O., Mazorchuk, V.: Classical finite transformation semigroups. An introduction. Algebra and Applications, Vol. 9, pp. xii+314. Springer, London (2009)

21. Green, J.: On the structure of semigroups. Ann. Math. 54, 163-172 (1951)

22. Grensing, A.-L.: Monoid algebras of projection functors. J. Algebra 369, 16-41 (2012)

23. Grensing, A.-L., Mazorchuk, V.: Categorification of the Catalan monoid. Semigroup Forum 89(1), 155-168 (2014)

24. Grensing, A.-L., Mazorchuk, V.: Categorification using dual projection functors. Preprint. arXiv:1501.00095. To appear in Commun. Contemp. Math

25. Hiller, H.: Geometry of Coxeter groups. Research Notes in Mathematics vol. 54, iv+213 pp. Pitman (Advanced Publishing Program), Boston (1982)

26. Humphreys, J.: Representations of semisimple Lie algebras in the BGG category $\mathcal{O}$. Graduate Studies in Mathematics, vol. 94, xvi+289 pp. American Mathematical Society, Providence, RI (2008)

27. Kazhdan, D., Lusztig, G.: Representations of Coxeter groups and Hecke algebras. Invent. Math. 53(2), 165-184 (1979)

28. Khovanov, M.: A categorification of the Jones polynomial. Duke Math. J. 101(3), 359-426 (2000)

29. Khovanov, M., Lauda, A.: A diagrammatic approach to categorification of quantum groups I. Represent. Theory 13, 309-347 (2009)

30. Kildetoft, T., Mazorchuk, V.: Parabolic projective functors in type A. Adv. Math. 301, 785-803 (2016)

31. Kildetoft, T., Mazorchuk, V.: Special modules over positively based algebras. Doc. Math. 21, 11711192 (2016)

32. Kildetoft, T., Mackaay, M., Mazorchuk, V., Zimmermann, J.: Simple transitive 2-representations of small quotients of Soergel bimodules. Preprint. arXiv:1605.01373

33. Kudryavtseva, G., Mazorchuk, V.: On multisemigroups. Port. Math. 72(1), 47-80 (2015)

34. Leinster, T.: Basic bicategories. Preprint. arXiv:math/9810017

35. Lusztig, G.: Introduction to quantum groups. Modern Birkhuser Classics, xiv+346 pp. Birkhäuser/Springer, New York (2010)

36. Mac Lane, S.: Categories for the Working Mathematician. Springer, Berlin (1998)

37. Mackaay, M., Mazorchuk, V.: Simple transitive 2-representations for some 2-subcategories of Soergel bimodules. J. Pure Appl. Algebra 221(3), 565-587 (2017)

38. Mackaay, M., Mazorchuk, V., Miemietz, V., Tubbenhauer, D.: Simple transitive 2-representations via (co)algebra 1-morphisms. Preprint. arXiv:1612.06325

39. Mackaay, M., Tubbenhauer, D.: Two-color Soergel calculus and simple transitive 2-representations. Preprint. arXiv:1609.00962

40. Mazorchuk, V., Miemietz, V.: Cell 2-representations of finitary 2-categories. Compos. Math. 147, 1519-1545 (2011)

41. Mazorchuk, V., Miemietz, V.: Additive versus abelian 2-representations of fiat 2-categories. Mosc. Math. J. 14(3), 595-615 (2014)

42. Mazorchuk, V., Miemietz, V.: Endmorphisms of cell 2-representations. Int. Math. Res. Notes 24, 7471-7498 (2016)

43. Mazorchuk, V., Miemietz, V.: Morita theory for finitary 2-categories. Quantum Topol. 7(1), 1-28 (2016) 
44. Mazorchuk, V., Miemietz, V.: Transitive 2-representations of finitary 2-categories. Trans. Am. Math. Soc. 368(11), 7623-7644 (2016)

45. Mazorchuk, V., Miemietz, V.: Isotypic faithful 2-representations of $\mathcal{J}$-simple fiat 2-categories. Math. Z. 282(1-2), 411-434 (2016)

46. Mazorchuk, V., Miemietz, V., Zhang, X.: Characterisation and applications of 7-split bimodules. Preprint. arXiv: 1701.03025

47. Mazorchuk, V., Zhang, X.: Simple transitive 2-representations for two non-fiat 2-categories of projective functors. Preprint. arXiv:1601.00097

48. Mazorchuk, V., Zhang, X.: Bimodules over uniformly oriented $A_{n}$ quivers with radical square zero. Preprint. arXiv: 1703.08377

49. Perron, O.: Zur Theorie der Matrices. Math. Ann. 64(2), 248-263 (1907)

50. Rouquier, R.: 2-Kac-Moody algebras. Preprint. arXiv:0812.5023

51. Rouquier, R.: Quiver Hecke algebras and 2-Lie algebras. Algebra Colloq. 19(2), 359-410 (2012)

52. Soergel, W.: The combinatorics of Harish-Chandra bimodules. J. Reine Angew. Math. 429, 49-74 (1992)

53. Soergel, W.: Kazhdan-Lusztig-Polynome und unzerlegbare Bimoduln über Polynomringen. J. Inst. Math. Jussieu 6(3), 501-525 (2007)

54. Stroppel, C.: Categorification of the Temperley-Lieb category, tangles, and cobordisms via projective functors. Duke Math. J. 126(3), 547-596 (2005)

55. Wald, B., Waschbusch, J.: Tame biserial algebras. J. Algebra 95(2), 480-500 (1985)

56. Xantcha, Q.: Gabriel 2-quivers for finitary 2-categories. J. Lond. Math. Soc. 92(3), 615-632 (2015)

57. Zhang, X.: Duflo involutions for 2-categories associated to tree quivers. J. Algebra Appl. 15(3), 1650041, 25 pp (2016)

58. Zhang, X.: Simple transitive 2-representations and Drinfeld center for some finitary 2-categories. Preprint. arXiv:1506.02402. To appear in J. Pure Appl. Algebra

59. Zimmermann, J.: Simple transitive 2-representations of Soergel bimodules in type $B_{2}$. J. Pure Appl. Algebra 221(3), 666-690 (2017)

60. Zimmermann, J.: Counting quasi-idempotent irreducible integral matrices. Preprint. arXiv:1701.03699 